\title{
Performance Evaluation of Proactive and Reactive Routing Protocols in Wireless Sensor Networks
}

\author{
Amit K. Kaushik \\ Department Of Computer science \\ MRCE, Faridabad, India
}

\begin{abstract}
In this paper, we study the two different type of routing strategies used in wireless sensor networks i.e. proactive and reactive routing mechanisms. These routing mechanisms are applied in different kind of applications for efficient routing in the network. In this paper, we have studied the different routing protocols which use these routing mechanisms and have compared them. We have also taken the homogenous and heterogeneous type of networks and also see the effect of homogeneity and heterogeneity on the routing in the network. So, we have taken LEACH and SEP routing protocols for homogenous and heterogeneous network respectively using proactive mechanism for routing and TEEN and TADEEC protocols for homogenous and heterogeneous using reactive mechanisms. Lastly we have compared the results of two routing strategies in term of stability period (the round in which first node dies), lifetime of the network and throughput i.e. data send to the base station per unit round.
\end{abstract}

\section{Keywords}

LEACH, SEP, TEEN, TADEEC etc

\section{INTRODUCTION}

Wireless sensor networks is an emerging research field where the research is continuously being done to make wireless sensor network better in terms of routing of the packets, the throughput of the network etc. This research is dominated by the fact that wireless motes has tiny amount of the resources in term of capacity like memory, processors etc and their deployment in the hostile places make them to be used in a very efficient way. [1] discussed the WSN stack layers and various research topics and challenges in routing in sensor networks. Like MANETs, the proactive and reactive routing strategies have also been developed for using in different applications in the wireless senor networks. The proactive and reactive routing mechanisms have been developed according to need of the network weather the traffic in the network is real time or not. As sometime the traffic is time bound and has to be reached within deadlines. In wireless sensor networks, the senor node has two processing elements i.e. sensing and processing elements. The sensing element is responsible for sensing the value for attribute in a particular environment. Suppose the sensor network is deployed in a forest for sensing the temperature for detection of a forest fire. Then the sensor node memory will store the attribute temperature and sensing element will sense the value of temperature and store in the temperature attribute. The processing element receives this data value from sensing element and processes it further. This processing is different in proactive and reactive routing. In proactive, the processing element send the data according to the TDMA slot allocated to the node by the cluster head (it is termed as report time in LEACH[2][3]).But in the reactive mechanism the hard and soft threshold determine the sending of the data value. In this there is no concept of report time but the data is sent according to its significance which is decided by the hard and soft threshold applied on the data. Section 2 give the research background, section 3 discusses the performance criteria and section 4 gives the simulation results and further discussions. Section 5 concludes the paper.

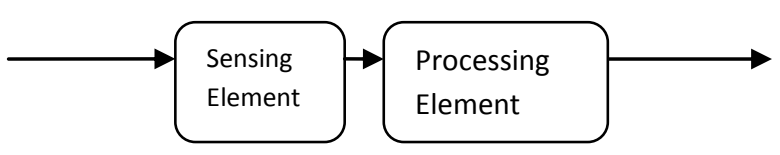

Fig 1: Showing the Sensing element and processing elements in sensor node.

\section{RESEARCH BACKGROUND}

\subsection{LEACH}

LEACH is a proactive routing protocol works in a homogenous network. This protocol uses clustering mechanism for routing in the network. The cluster head for each cluster head is chosen based on the expression as

$$
T(i)=p /(1-p(\operatorname{rmod} 1 / p))
$$

Here the $\mathrm{p}$ is probability of a node $\mathrm{n}$ getting elected as cluster head and $\mathrm{r}$ is round number. A random no $\mathrm{f}$ is chosen between $[0,1]$ and it is compared with Threshold $T(n)$ and if $T(n)$ is greater than $\mathrm{f}$ then that node become cluster head otherwise not. The leach sense the data and send the data to the cluster head based on the TDMA slot allocated by cluster head the cluster head then send the data to the basestation. CDMA is used in the LEACH to prevent intercluster interference. All the cluster based routing protocols used the LEACH protocol for cluster head selection process. PEGASIS [4] is also proactive protocol that uses chaining technique for transmission of the data without formation of clusters in the network. Several improvements of LEACH came in [5] and [6] which removed the drawbacks of LEACH.

\subsection{EDEEC}

EDEEC [7] is a proactive protocol used in heterogeneous network. It is an extension of SEP [8] and DEEC [9]. DDEEC [10] is also extension which improved the DEEC further. But [11] has evaluated the performance of improvements of DEEC where it found that EDEEC is better than DDEEC. EDEEC protocol used three types of nodes i.e. normal, advanced and super nodes. These nodes have energies in increasing order i.e. normal, advanced and super respectively and the probabilities of getting elected as cluster head follow the reverse order i.e. super, advanced and normal respectively. Thus higher energy nodes will increase the epoch time for less energy node thus finally increase the stability period of the network. This follows the same procedure for cluster head election and sending of the data as of the LEACH. The nodes are distributed according to $\mathrm{m}$ and $\mathrm{mo}$ as

normal $=n *(1-m)$;

advance $=n * m *(1-m o)$; 
super $=n * m * m o$;

And their energies are:

normal: $\quad E_{0}$

advanced: $(1+a) E_{0}$

super: $\quad(1+b) E_{0}$

$p_{i}=\left\{\begin{array}{l}\frac{p_{o p t} \times E_{i}(r)}{(1+m(a+m o . b)) \times \bar{E}(r)} \text { if node is normal node } \\ \frac{p_{o p t} \times E_{i}(r) \times(1+a)}{(1+m(a+m o . b)) \times \bar{E}(r)} \text { if node is advanced node } \\ \frac{p_{o p t} \times E_{i}(r) \times(1+b)}{(1+m(a+m o . b)) \times \bar{E}(r)} \text { if node is super node }\end{array}\right.$

and the thresholds for cluster head selection is calculated as below:

$T(i)=\left\{\begin{array}{cc}\frac{p_{\text {norm }}}{1-p_{\text {norm }}\left(r \times \bmod \left(\frac{1}{p_{\text {norm }}}\right)\right)} & \text { if } i \text { is normal and } i \in G \\ \frac{p_{\text {adv }}}{1-p_{\text {adv }}\left(r \times \bmod \left(\frac{1}{p_{\text {adv }}}\right)\right)} & \text { if } i \text { is advanced and } I \in G \\ \frac{p_{\text {sup }}}{1-p_{\text {sup }}\left(r \times \bmod \left(\frac{1}{p_{\text {sup }}}\right)\right)} & \text { if } i \text { is super and } I \in G, \\ 0 & \text { otherwise }\end{array}\right.$

Where G, G' and G" are sets of nodes which have not become cluster heads till ate specific round number $r$.

\subsection{TEEN}

TEEN [12] is a reactive routing protocol used in homogenous network. This protocol is used in deadline specific network traffic. It uses two thresholds hard and soft thresholds. These thresholds decide whether to send the data to the cluster head or not. This protocol is based on different observations. First is that the nodes in the cluster sense similar type of data so cluster head get overwhelm with similar type of data. Second is that a node send the data to its cluster head at regular slots without knowing that it might send the same or similar data before this slot. Thus it is wastage of energy as node waste a lot of energy in transmission of the data. Also suppose data has to be sent within a time deadline but node would send data only on the report time or slot given. So TEEN is effective in real time application where the packet sending and receiving is timely bound. A sensed value is significant when it is greater than hard threshold and difference between sensed value and previous sent value should be greater than soft threshold. The TEEN can be applied on both sensor node and cluster head. A node deployed with TEEN protocol sense the data and store in a current sensed variable and it compare with hard threshold. If current sensed value is greater than the hard Threshold then the difference of the current sensed data and previous sent value(which would become the sensed value and stored in sensed_value) is taken. The difference shows the data importance as the soft threshold is taken according to the application and soft threshold shows that how much value difference from previous sent value is taken that would be regarded as significant and nodes will switch on their transmitter to transmit and basestation their receiver. Here we have taken hard threshold as 100 and soft threshold as 2 .

\subsection{TADEEC}

TADEEC [13] protocol is made to work in a reactive mode in a heterogeneous network. So it basically used the best aspects of both reactive routing mechanism and heterogeneity of the network. It is extension of EDEEC with four types of nodes i.e. normal, advanced, super and superadvanced with their increasing energies. It uses same strategy as used in LEACH for cluster head selection. The probabilities of getting elected as cluster head decreases from superadvanced to normal. This apply optimization protocol TEEN in the node to work in a reactive mode in the network. The node distribution is done according to $\mathrm{m}$ and $\mathrm{m} 0$ and their energies are as :

$$
\begin{aligned}
& \text { normal }=(1-m) * n ; \\
& \text { advanced }=(1-m 0) * m * n ; \\
& \text { super }=(m 0 * m * n) / 2 ; \\
& \text { supadvanced }=(m 0 * m * n) / 2 ; \\
& \text { normal nodes: } E_{0} \quad \text { advanced: } E_{0}(1+a) \\
& \text { super: } E_{0}(1+b) \quad \text { superadvanced: } E_{0}(1+c) \\
& \text { where } a=c / 2 ; b=3 c / 4 ; c=1 ;
\end{aligned}
$$

The probabilities for election of cluster head for normal, advanced, super and superadvanced nodes are as:

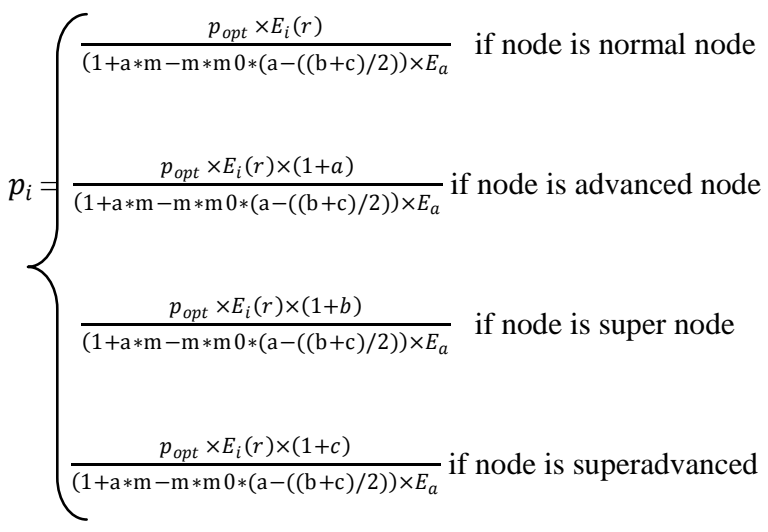

and the thresholds calculation is done as:

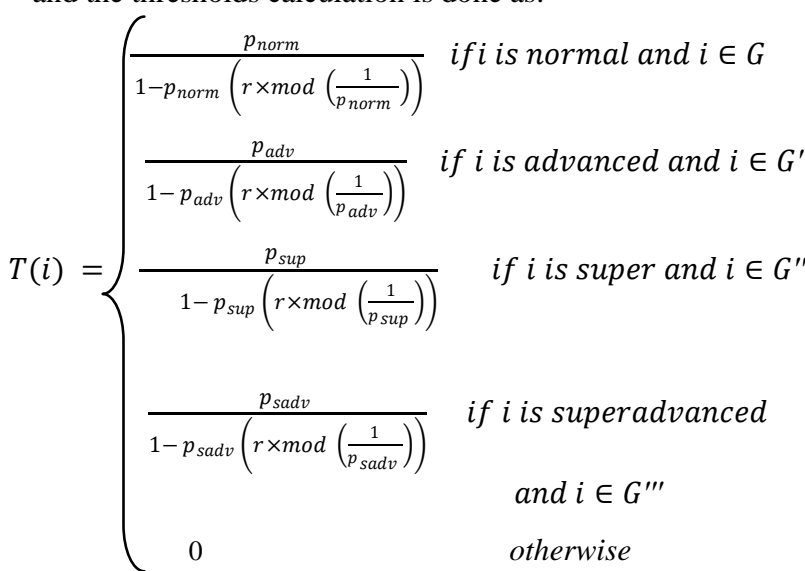

The $p_{\text {norm }}, p_{a d v}, p_{\text {sup }}$ and $p_{\text {sadv }}$ are probabilities for getting selected as cluster head for normal ,advanced, super and superadvanced nodes.G,G',G', and G',' are set of nodes which have not become cluster heads till round where $r$ is round no. 


\section{PERFORMANCE CRITERIA}

Different protocols are compared taking four parameters

1.Stability period:

This time is that round number in which first node dies in an network dies.

\section{Dead nodes:}

These are number of dead nodes get died till last round.This parameter shows how long a network exists for transfering of data to the basestation. So lesser will be dead nodes better will be network performance.

\section{Lifetime of the network using Alive Nodes:}

These are nodes which exists till the last round.So higher would be the alive nodes better will network perform.

\section{Throughput(or Data sent to basesation per round):}

This measures the data sent per round to the basestation by the cluster head.A good protocol will have high throughput.

\section{SIMULATIONS AND DISCUSSIONS}

In this section we simulated various routing protocols using proactive and reactive mechanisms. These simulations are done in matlab. Various Protocol simulation parameters are given in table 1 .We have simulated a forest environment where sensors are deployed at random location around a basestation to detect the forest fire. So sensor nodes sense the temperature from their adjacent environment. The sink (or basestation) is located at middle of the forest environment. The forest is taken an area of $100 * 100$ meters square. The different simulations are done and their results are collected. These data are collected according to different values heterogeneity level taking different in different simulations. So in this forest environment we have taken the hard threshold in TEEN and TADEEC as 100 degree Celsius so temperature above 100 degree Celsius is significant. And also soft threshold is taken as 2 so current sensed value should be differ from previous sent value by 2 only then it would be sent to the cluster head. The simulation is done in different environments. Different scenarios for doing comparison are given below as:

1.Homogenous network having proactive mechanism for doing routing.Eg. $\mathrm{LEACH}$

Table 1: lists of simulation parameters

\begin{tabular}{|l|l|}
\hline Parameters & Value \\
\hline Area & $100 * 100$ square meters \\
\hline Basestation location & $(50,50)($ in m) \\
\hline Initial Energy & $0.5 \mathrm{~J}$ \\
\hline Transmission Energy & $50 \mathrm{~nJ} / \mathrm{bit}$ \\
\hline Receiver Energy & $50 \mathrm{~nJ} / \mathrm{bit}$ \\
\hline No of Nodes & 100 \\
\hline Free space Amplification Energy & $10 \mathrm{pJ} / \mathrm{bit} / \mathrm{m}^{2}$ \\
\hline
\end{tabular}

\begin{tabular}{|l|l|}
\hline Multipath Amplification Energy & $0.0013 \mathrm{pJ} / \mathrm{bit} / \mathrm{m}^{4}$ \\
\hline Message Size(B) & $4000 \mathrm{bits}$ \\
\hline Maximum Round & 20000 \\
\hline Aggregation Energy & $5 \mathrm{~nJ} / \mathrm{bit} /$ packet \\
\hline Hard Threshold(for reactive $\mathrm{n} / \mathrm{w})$ & 100 degree Celsius \\
\hline Soft threshold(for reactive $\mathrm{n} / \mathrm{w})$ & 2 degree Celsius \\
\hline$p_{\text {opt }}$ & 0.1 \\
\hline $\mathrm{m}$ ( for heterogeneous network) & 0.5 and 0.6 \\
\hline m0(for heterogeneous network) & 0.2 and 0.4 \\
\hline
\end{tabular}

2. Homogenous network having reactive mechanism for doing routing. Eg. TEEN

3. Heterogeneous network having proactive mechanism for doing routing. Eg. EDEEC

4. Heterogeneous network having reactive mode of routing. Eg. TADEEC.

These protocols are studied and compared in terms of stability period, lifetime and throughput of the network. The simulation parameters are listed in the Table 1 .The protocols are simulated in the environment of 100 nodes in the area of 20000 meters squares. The leach protocol is deployed in a homogenous environment with only normal nodes. The TEEN protocol is taken in homogenous environment with normal nodes but working in the reactive mode. The EDEEC work in heterogeneous mode with normal, advanced and super nodes

and work in proactive mode. The TADEEC is working in the reactive mode in the heterogeneous network with normal, advanced, super and super advanced nodes. The EDEEC work in heterogeneous mode with normal, advanced and super nodes and work in proactive mode. The TADEEC is working in the reactive mode in the heterogeneous network with normal, advanced, super and super advanced nodes. The output of each protocol is shown in the tabular and graphical form. The comparison of different protocols taking different parameters is:

\subsection{Stability Period}

The Figure 2 and 4 shows the dead nodes in the network for LEACH, TEEN, EDEEC and TADEEC for different heterogeneity. In leach the first node get died before round number 500. While other protocols EDEEC and TEEN have stability period between 1000 and 1500 in most of the simulations. The TADEEC has highest stability period above 3000 rounds. It can be observed that the TADEEC is performing well over protocols. The stability period of proactive protocol LEACH is least. But the stability period of reactive protocol TEEN is less than proactive protocol EDEEC. This means heterogeneity increases the stability period as it increases the epoch times for the less energy nodes in the network irrespective of the proactive and reactive mode. TADEEC increases the heterogeneity further in EDEEC protocol but it works in reactive mode and performs well than other protocol. So this can be said that heterogeneity level in the sensor nodes improves the network but working in 
a reactive mode also gives advantage to the network. These both factors improve the communication and save the energies of the nodes and increases the stability period.

\subsection{Lifetime}

The Alive nodes are shown in figure 3 and 5 for different protocols for different heterogeneity. The lifetime for LEACH is lesser than TEEN in all the cases. The TADEEC has the highest lifetime among all protocols. LEACH deployed network has shorter life with all nodes get died before 4000 rounds in most simulations but it also performed better than TEEN in some simulations. EDEEC has outperformed both LEACH and TEEN with lifetime around 5000 rounds. However the TADEEC has highest lifetime with lifetime even greater than 10000 rounds in some simulations.

\subsection{Throughput}

In reactive protocols such as TEEN and TADEEC, the data is collected by the sensor node and then after applying the hard and soft thresholds the data is sent to the cluster heads. Then it is up to cluster head whether to apply the hard and soft thresholds or not, the cluster head sent the data to the basestation. In proactive protocols such as LEACH and EDEEC, the data is sent at slotted timing given in TDMA schedule. The data sent per round is compared in the figure 6 . The data sent to the basestation i.e. Fig. 8 is used to determine the throughput of the protocols. The data is least in LEACH while maximum in TADEEC. The data sent is less in TADEEC in initial rounds but become stable in last rounds and packets sent become more than that of EDEEC in last rounds. The throughput of the TADEEC is maximum i.e. 14 packets are sent in one round where LEACH has least i.e.1 packet per round.

The Analysis based on above results can be described as:

1. The specific protocol for deadline-specific (or real time network) is required as it needs special attention but LEACH does not perform well in real time network. So we can say reactive protocols perform better in those environment having real time delivery problems. Also these reactive networks save a lot of energy by using thresholds specific transmission and they don't have to send control packets regarding the TDMA schedule.

2. The Heterogeneity in the network is useful for both deadline specific and non-deadline specific network as this decreases the chances of the less energy nodes to become cluster heads frequently in the network. So EDEEC outperforms TEEN in term of all parameters in both kind of environments i.e. deadline or non-deadline environments. Also when we increase the heterogeneity in the network then it also effects the stability period, lifetime and packets transmission in the network.

3. The Combining Aspects of heterogeneity and threshold constrained transmission make the network live longer, have a high stability period and have a high throughput. The TADEEC protocol uses this property and outperforms all other protocol.

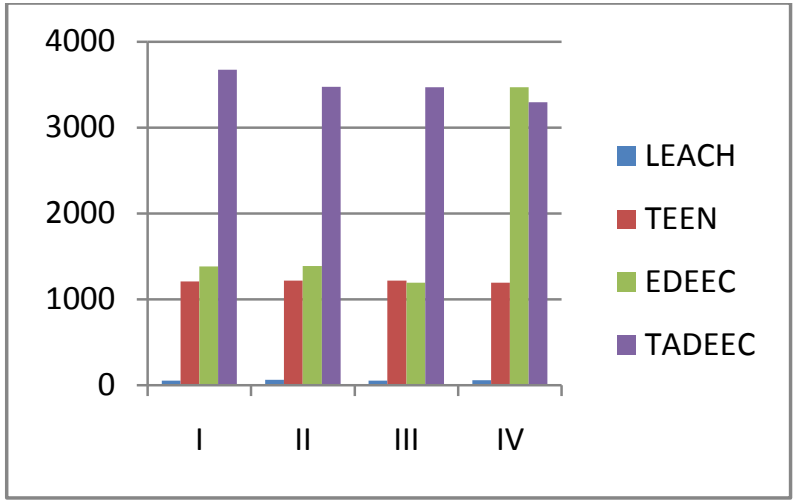

Fig 2: Showing the Stability Period of different Protocols when $\mathrm{m}=0.5$ and $\mathrm{m} 0=0.2$.

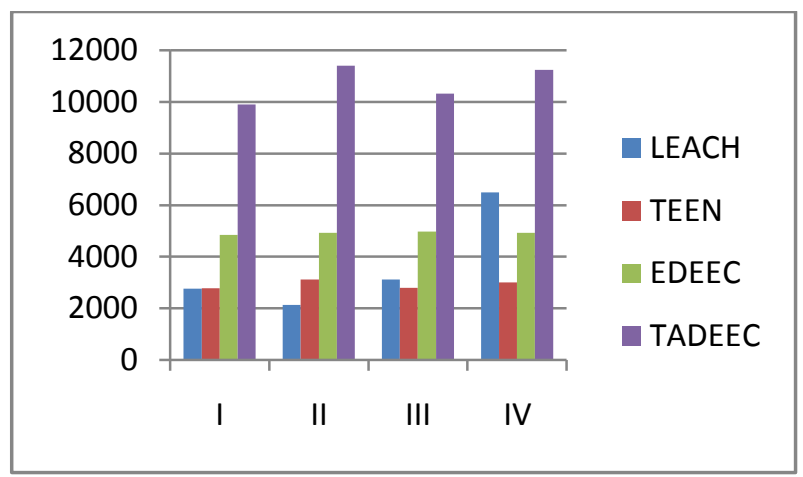

Fig 3: Showing the Lifetime period of different Protocols when $\mathrm{m}=0.6$ and $\mathrm{m} 0=0.4$.

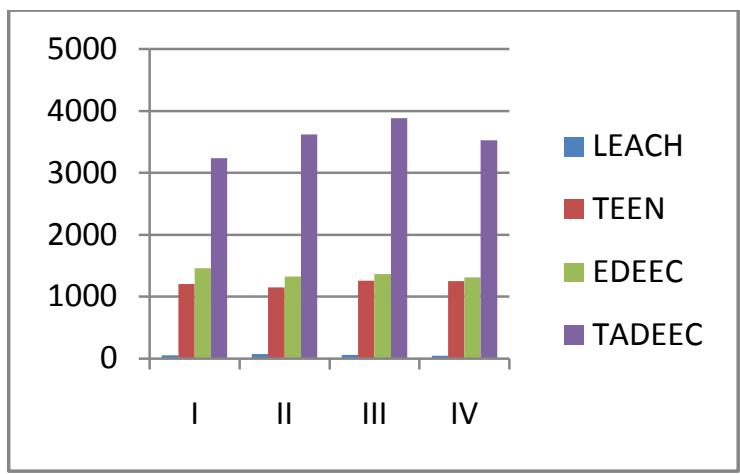

Fig 4: Showing the stability period of different Protocols when $\mathrm{m}=0.6$ and $\mathrm{m} 0=0.4$.

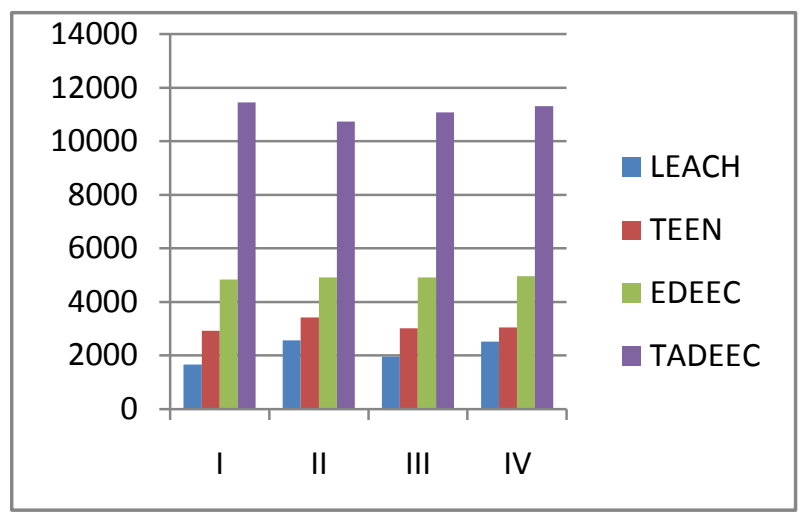

Fig 5: Showing the Lifetime period of different Protocols when $\mathrm{m}=0.6$ and $\mathrm{m} 0=0.4$. 


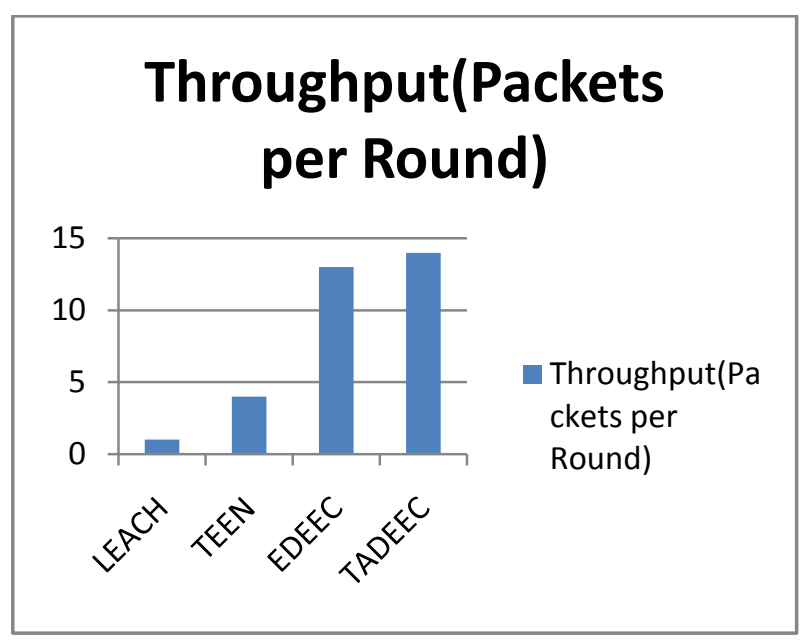

Fig 6: Showing the throughput of the network for different protocols $\mathrm{m}=0.5$ and $\mathrm{m} 0=0.2$.

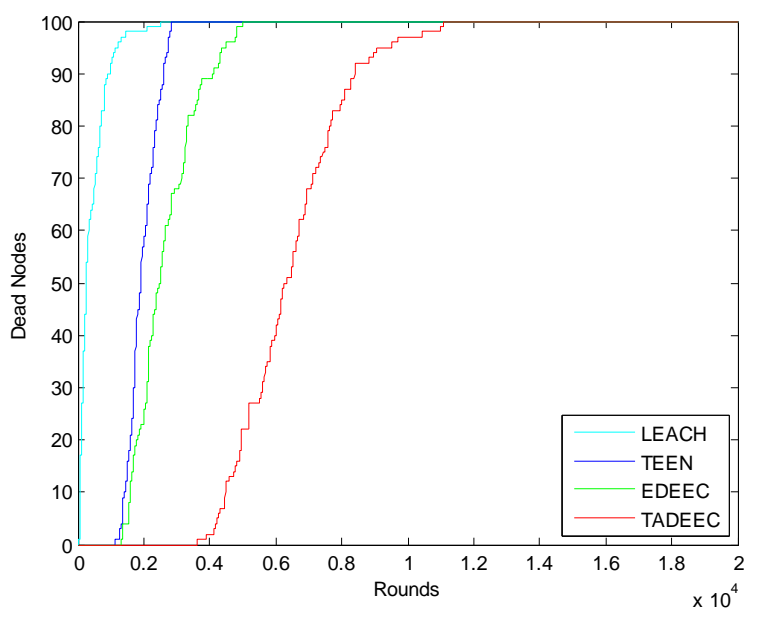

Fig 7: Showing the Dead nodes in the network for different protocols.

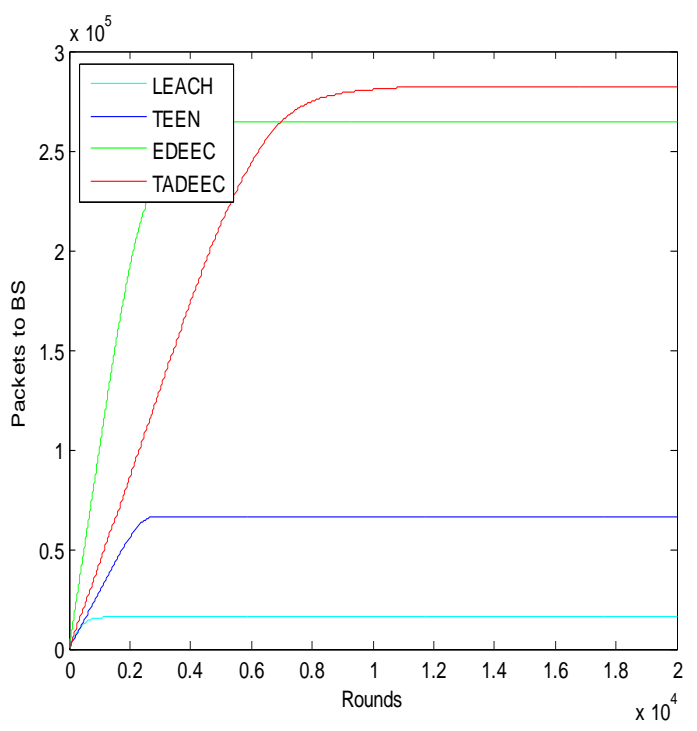

Fig 8: Showing the data sent to the basestation by the cluster heads.

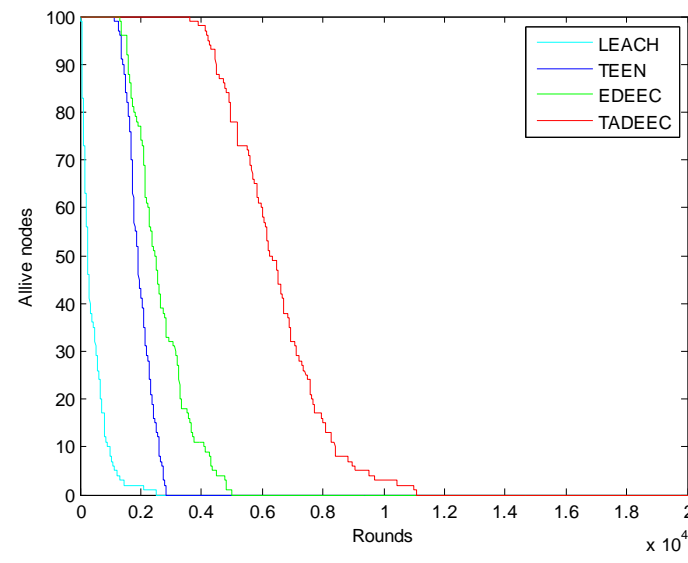

Fig 9: Showing the Alive nodes in the network for different protocols.

\section{CONCLUSION AND FUTURE SCOPE}

This paper is a performance evaluation and comparative study of proactive and reactive protocols in homogenous and heterogeneous environment. In this we have found that the real time processing requires special protocol and $\mathrm{LEACH}$ cannot be preferred in this environment. But in some environment which does not require real time processing LEACH is better than TEEN. Also the heterogeneous aspect makes EDDEC protocol even better than TEEN. The TEEN also saves a lot of time and space as it does not require framing of TDMA schedule. But it needs to be updated according to the environment as the thresholds i.e. hard and soft thresholds have to be updated. The network performs best when heterogeneous sensors in the network are deployed with thresholds specific transmission property. Thus we can say that TADEEC is best suited as it combines the best of both approaches i.e. heterogeneous and thresholds constrained network. In future we will try to investigate other factors responsible for making network more reliable, having better lifetime etc. In all protocols we studied had a problem of nonoptimal distribution of the cluster heads and nodes. So we will also consider contribution of how optimal distribution of cluster heads and nodes affect the life, stability period and throughput in addition to threshold specific transmission and heterogeneous nature of the nodes factors. Also we take into account the non-clustering effect on performance of the network parameters.

\section{REFERENCES}

[1] F. Akyildiz, W. Su, Y. Sankarasubramaniam, and E. Cayirci, "A survey on sensor networks," IEEE Communications Magazine, vol. 40, no. 8, pp. 102-114, 2002.

[2] W.Heinzelman, A. Chandrakasan, and H. Balakrishnan, "Energy-efficient communication protocol for wireless sensor networks," in the Proceeding of the Hawaii International Conference System Sciences, Hawaii, January 2000.

[3] W.R. Heinzelman, A.P. Chandrakasan, H. Balakrishnan, "An applicationspecific protocol architecture for wireless microsensor networks", IEEE Transactions on Wireless Communications 1 (4) (2002) 660-670.

[4] Lindsey S,Raghavenda CS.PEGASIS : Power efficient gathering in sensor informationsystems[C].Williamson 
DA.Proc of the IEEE Aerospace Conf.New York:IEEE Press, 2002:1125-1130.

[5] S. H. Gajjar, K. S. Dasgupta, S. N. Pradhan, K. M. Vala "Lifetime Improvement of LEACH Protocol for Wireless Sensor Network" In INTERNATIONAL CONFERENCE ON ENGINEERING, NUICONE-2012, 06-08DECEMBER, 2012.

[6] Rupesh Mehta, Abhishek Pandey\& Pratik Kapadia"Reforming Clusters Using C-LEACH in Wireless Sensor Networks"In International Conference on Computer Communication and Informatics (ICCCI 2012), Jan. 10 - 12, 2012, Coimbatore, INDIA.

[7] Parul Saini, Ajay.K.Sharma, "E-DEEC- Enhanced Distributed Energy Efficient Clustering Scheme for heterogeneous WSN", in: $20101^{\text {st }}$ International Conference on Parallel, Distributed and Grid Computing (PDGC - 2010).

[8] G. Smaragdakis, I. Matta, A. Bestavros, "SEP: A Stable Election Protocol for clustered heterogeneous wireless sensor networks", in: Second International Workshop on Sensor and. Actor Network Protocols and Applications (SANPA 2004), 2004.

[9] L. Qing, Q. Zhu, M. Wang, "Design of a distributed energy-efficient clustering algorithm for heterogeneous wireless sensor networks". ELSEVIER, Computer Communications 29, 2006, pp 2230- 2237.

[10] Elbhiri, B. , Saadane, R. , El Fkihi, S. , Aboutajdine, D. "Developed Distributed Energy-Efficient Clustering (DDEEC) for heterogeneous wireless sensor networks", in: 5th International Symposium on I/V Communications and Mobile Network (ISVC), 2010.

[11] T. N. Qureshi, N. Javaid, M. Malik, U. Qasim, Z. A. Khan "On Performance Evaluation of Variants of DEEC in WSNs"In Seventh International Conference on Broadband, Wireless Computing, Communication and Applications 2012.

[12] A. Manjeshwar and D. P. Agarwal, "TEEN: a routing protocol for enhanced efficiency in wireless sensor networks," In 1st International Workshop on Parallel and Distributed Computing Issues in Wireless Networks and Mobile Computing, April 2001

[13] Anamika Chauhan and Amit Kaushik, "TADEEC : Threshold Sensitive Advanced Distributed Energy Efficient Clustering Routing Protocol for Wireless Sensor Networks," International Journal of Computer Applications (0975 - 8887),Volume 96 No.23 June 2014.

[14] MATLAB 7.4.0(R2007) www.mathworks.com 